\title{
Effects of Free Range-of-Motion Upper Limb Exercise Based on Mirror Therapy on Shoulder Function in Patients After Breast Cancer Surgery: Study Protocol for a Randomized Controlled Trial
}

\section{Ru Zhen Yuan}

Shanghai University of Traditional Chinese Medicine

\section{Kun Peng Li}

Shanghai University of Traditional Chinese Medicin

\section{Xiao Lin Wei}

Shanghai University of Traditional Chinese Medicine

\section{Wei Zheng}

Longhua Hospital Shanghai University of Traditional Chinese Medicine

Yi Ye

Fudan University Zhongshan Hospital

\section{Ming Yue Wang}

Shanghai University of Traditional Chinese Medicine

Jie Ting Jiang

Shanghai University of Traditional Chinese Medicine

Caiqin Wu ( $\triangle$ yuwuchina@aliyun.com )

Shanghai university of traditional Chinese medicine https://orcid.org/0000-0002-9420-8958

\section{Study protocol}

Keywords: Breast cancer survivors, free range-of-motion upper limb exercise, mirror therapy, shoulder function

Posted Date: September 13th, 2021

DOl: https://doi.org/10.21203/rs.3.rs-248716/v1

License: (c) (1) This work is licensed under a Creative Commons Attribution 4.0 International License. Read Full License 
Version of Record: A version of this preprint was published at Trials on November 17th, 2021. See the published version at https://doi.org/10.1186/s13063-021-05789-2. 


\section{Abstract}

Background Shoulder function complications are common after surgery for breast cancer. Quite a few survivors still reported a limited shoulder range of motion, even though the free range-of-motion upper limb exercise is helpful to restore shoulder function post-operatively. Mirror therapy(MT) is a classical and effective rehabilitation technique to recover motor and sensory function for limbs, in addition, studies have reported that MT has the influence on patients with shoulder functional dysfunction including increasing shoulder range of motion, improving shoulder function scores, and decreasing pain scores. Here we describe a protocol of a randomized controlled trial to explore if the free range-of-motion upper limb exercise based on mirror therapy has efficacy on shoulder function in breast cancer survivors after surgery.

Methods/design This is a prospective, single-blind, two-arm randomized controlled trial. An estimated 70 participants will be randomly allocated to 1) MT group or 2) control group. The participants in the control group receive free range-of-motion upper limb exercise, and participants in the MT group will engages in free range-of-motion upper limb exercise based on mirror therapy. The intervention will start from the first day after surgery and will be completde at 8 weeks after surgery. Primary outcomes in this protocol is shoulder range of motion(ROM), while the Constant-Murley Score(CMS), Disability of arm, shoulder, and hand Questionnaire (DASH), Tampa Scale of Kinesiophobia (13-item TSK), Visual Analogue Scale(VAS), grip strength, arm circumference, and lypmphedema are secondary outcomes. Assessment will be conducted before allocation(baseline) and at 2 weeks, 4 weeks, and 8 weeks after surgery.

Discussion Based on results that mirror therapy has influence on shoulder function immediately after intervention in patients without nerve injury, this randomized controlled trial is the first study to observe the efficacy of mirror therapy on shoulder function after a long time intervention in breast cancer survivors. We look forward the innovation of this study for both breast cancer rehabilitation and mirror therapy.

Trial registration Chinese Clinical Trial Registry (ChiCTR): ChiCTR 2000033080. Registered on 19 May 2020, http://www.chictr.org.cn/searchproj.aspx.

\section{Background}

Breast cancer(BC)is the most frequently diagnosed cancer among women throughout the world ${ }^{1}$, however, the five-year survival for breast cancer is the highest among women's cancer,besides, it is going up gradually ${ }^{2}$. Since both prevalence and survival rates continue to rise, breast cancer tends to become chronic. Similar to patients with chronic diseases, breast cancer survivors have to fight with the treatment-related complications that affect their health status and quality of life. Shoulder function complications are one of the most common sequelae following treatment for breast cancer, which can manifest soon after surgery or would appear many years later. Typical symptoms include a restricted shoulder range of motion, stiffness and weakness of the shoulder, and pain ${ }^{3}$. It is reported a prevalence 
rate of $32.9 \%$ of a restricted shoulder range of motion in patients at 1 year after axillary lymph node dissection, and a prevalence rate of $23.2 \%$ in patients at 1 year after sentinel lymph node biopsy ${ }^{4}$. Pain and numbness are found of $51.1 \%$ in breast cancer survivors at 2 years after breast cancer surgery ${ }^{5}$. Shoulder function complications will adversely affect breast cancer survivors in daily activity ability, medical burden, social life, and quality of life. It is limited to follow-up time, the shoulder function complications persist much longer than it is reported in many studies. Hauerslev reported $25.7 \%$ and $30 \%$ breast cancer survivors who were evaluated 11.7 years after surgery have a decreased shoulder mobility and shoudler pain respectively ${ }^{6}$. In the long run, shoulder function complications will damage the physical, psychosocial and social domains of the function of breast cancer survivors, thus it is still a matter of concern.

Shoulder function complications of breast cancer are caused primarily by surgery, which causes defect and damage of soft tissues and myofascial restrictions on the upper body, in the effect of scar tissue formation, postsurgical pain, protective posturing, or breast reconstruction, chest wall soft tissue will become tighten and shorten, losing their gliding ability that is relative to shoulder joint ${ }^{3,7-9}$. Besides, radiotherapy probably causes soft-tissue fibrosis and radiation dermatitis, leading to shoulder pain and dysfunction. Other complications such as wound complications, lymphedema, and axillary web syndrome, on the other hand can also induce shoulder function complications ${ }^{10-13}$.

Free range-of-motion upper limb exercise following breast cancer treatment can improve shoulder range of motion and decrease chest tightness by moving and stretching the affected arm to restore shoulder joint flexibility and increase scar-plasticity ${ }^{14,15}$. Despite the free range-of-motion upper limb exercise can improve long-term gains in shoulder function without increasing the wound complication, there remains underutilization of free range of motion upper limb exercise, quite a few breast cancer survivors still have a restricted shoulder range of motion and report shoulder pain even within 6 months after treatment ${ }^{16,17}$. Many survivors fear of movement after surgery, because they fear of the affected arm injury. According to the cognitive model of fear of movement/(re) injury of Johan Vlaeye, individual fears of (in)injury will lead to fear-avoid behaviors in daily activity ${ }^{18}$. Studies have reported that fear of the affected arm injury is one of the common barriers to underutilization of rehabilitation for breast cancer survivors ${ }^{16,17}$.

Two prospective studies have suggested that mirror therapy(MT) may be a useful tool to improve shoulder function in patients with a restricted shoulder range of motion and shoulder pain. MT is a classic rehabilitation therapy based on visual feedback of plane mirrored images. Placing a mirror along the midsagittal plane between the two limbs, in a position with the affected limb behind the mirror and the unaffected limb in front of the mirror, in this way, the reflection of the limb in front of the mirror is always superimposed on the limb hidden behind the mirror so that it could create a visual illusion of the health and intactness of the contralateral limb. The patient moves his/her limbs simultaneously while he/she needs to lean forward slightly to watch and concentrate on complete reflection of the unaffected limb in the mirror and think of reflection as the moving of the affected limb behind the mirror. MT is always a useful rehabilitation technique for patients with nerve injury, such as storks and phantom limb 
pain ${ }^{18-21}$. In addition, a few,studies have reported that MT can improve limb motor function in patients without nerve injury. Altschuler reported a patient who has a fractured wrist but no neurovascular impairment can extend her wrist actively after 10 weeks MT intervention ${ }^{22}$. Rosen showed MT improves a woman's fingers active flexion, whose fingers were bit by a cat. In addition, a patient with rheumatoid arthritis gain good coordination and flexibility of fingers after 8 weeks MT intervention ${ }^{23}$.

The details of two studies of MT intervention on shoulder are as follows. Louw conducted a cohort study, 69 patients with shoulder pain and limited range of motion were asked to move their shoulder in front of a mirror 10 times for 3 minutes. Immediate assessment after intervention showed that shoulder flexion ROM has a mean increase of $14.5^{\circ}$, and pain levels averagely decreased $0.48^{24}$. Baskaya has conducted an experiment of MT in patients with adhesive capsulitis. This is a two-arms randomized controlled trial, the experimental group was administered to do shoulder movement with the reflecting side of the mirror, while the control group was administered to do exercise with the non-reflecting side. Immediate measurement after intervention showed that shoulder ROM, VAS score, and The University of CaliforniaLos Angeles (UCLA) shoulder score of the experimental group improved greatly than that of the control group $^{25}$.

Potential mechanism of MT on motor recovery is using a mirrored image of the intact limb to compensate proprioceptive feedback to a motor command originated by the affected limb, in other words, MT normalizes the efference-afference loop of the sensorimotor brain circuits in a movement process ${ }^{26,27}$. In this process, visual information primarily influence the proprioception. Studies in health individuals found the position of hand in the mirror can make a biase of the position of hand behind a mirror. Louw and Baskaya measure the outcome immediately after intervention, thus their study results only showed MT has a influence on shoulder movement in patients without nerve injury. However whether MT can improve shoulder function in a long time intervention needs advanced study. Based on current studies, given that advantages of low-cost, practical, and easy-to-apply, we are interested in the efficacy of MT on shoulder function in breast caner survivors. In addition, to our knowledge, no study have applied MT on shoulder function in breast cancer survivors.

\section{Objectives}

The purpose of this prospective, explorative randomized controlled trial is that if free range-of-motion upper limb exercise based on MT could bring gains on efficacy compared with effects of free range-ofmotion upper limb exercise on shoulder function in patients after breast cancer surgery. The main purpose of the trial are as follows:

1. Observing the efficacy of free range-of-motion upper limb exercise based on MT on shoulder range of motion in breast cancer survivors.

2. Observing the effect of free range-of-motion upper limb exercise based on MT on shoulder function score, shoulder pain,and upper limb function and disability in breast cancer survivors. 
3. Observing the effect of free range-of-motion upper limb exercise based on MT on fear of movement in breast cancer survivors.

\section{Methods}

\section{Design}

This is a prospective, single-blind, two-arm randomized controlled trial that will explore the effect of free range-of-motion upper limb exercise based on MT on shoulder function in patients after breast cancer surgery. An estimated 70 participants from the galactophore department will be randomly allocated to 1 ) MT group or 2) control group. The control group is administrated to do free range-of-motion upper limb exercise, while the MT group engages in free range-of-motion upper limb exercise based on MT. A intervention team consisting of nurses, rehabilitation therapists, and surgeons will be set up. Outcomes will be measured at baseline, 2 weeks, 4 weeks, and 8 weeks after surgery. Fig. 1 shows the Recommendations for Intervention Trials (SPIRIT) flow diagram for this study.

\section{Ethical}

The ethical approval of this study was approved by The Ethical Committee of Longhua hospital affiliated to the Shanghai University of traditional Chinese medicine on 5 May 2020(reference number:

2020LCSY016). The research scheme, case report form, and informed consent form were approved by the Ethics Committee. Informed consent will be obtained from all participants before the intervention.

\section{Study setting}

The study will be carried out at galactophore department in the two hospitals in Shanghai, they are Longhua hospital Shanghai University of Traditional Chinese Medicine and Fudan University Zhongshan Hospital. Outcome measurement will be conducted at a separate quiet room in the hospital.

\section{Sample size}

The primary outcome in this study is shoulder range of motion, which is the main effect indicator for sample size estimating in this randomlized controlled trial. Referring to the Cohen's effect size value of 1.3799 in the study of Ba,skaya, to achieve $95 \%$ power and 0.05 error range, 30 of the total sample size can be calculated using the G-power 3.1 software. However, a higher number of the sample size was planned to be used in this trial.because of repeated measurement. We used GLIMMPSE software for repeated measurement calculation with the shoulder flexion range on motion as the main effect indicator that refers to the result of $\mathrm{Ba}$,skaya.We choose a test power of 0.95 and type one error rate of $0.05,58$ of sample size was calculated, considering $20 \%$ percent dropout rate, the total sample size is about 70 .

\section{Participants}

Inclusion criteria 
1. Women aged $\geq 18$ years;

2. Clinically diagnosed as breast cancer for the first time according to imaging examination and (or) pathological examination pre-operation;

3. The treatment of breast includes modified radical mastectomy, mastectomy, or conservation surgery, the treatment of the axilla includes axillary lymph nodes dissection(ALND) or sentinel lymph node biopsy(SLNB);

4. Having ability to use social software such as WeChat;

5. The signing of the informed consent form;

Exclusion criteria

1. Clinically diagnosed bilateral breast cancer, or cancer metastasis;

2. With shoulder disease, upper limb fracture, neurological deficits, lymphangitis, and any injury of the upper limb;

3. Have suffered from severe cardiovascular disease, cognitive limitations, mental disease;

4. Visual field defect;

5. Receive immediate reconstruction in surgery;

Elimination criteria

1. The type of surgery not meet the inclusion criteria because of type of surgery would changed during

operation.

Drop out criteria

1. Withdrawing from trial voluntary;

2. Pass away during the trial;

3. Lose contact such as remove the residence or change the hospital

\section{Recruitment}

We will recruit eligible participants began on 1 July 2020 and will be completed in June 2021. According to the fixed date of admission of breast cancer patients in two hospitals, we regularly recruit participants at Fudan University Zhongshan Hospital every Monday, and at the other hospital every Thursday. The primary researcher is responsible for recruitment and can access the medical histories of patients after getting the permission of patients. We will provide pictures, relevant materials, and informed consent to introduce the program and the purpose of the trial, telling subjects the advantages and disadvantages of the intervention. We reconfirm and determine the participant of her eligibility according to 
inclusion/exclusion criteria. Eligible participants who signed the informed consent will be finally recruited.

\section{Randomization and blinding}

Random-number sequences are generated in randomization software by an independent researcher, the 200000 was used as the seed number to generate70 Random-numbers. For avoiding tampering or transillumination, information of trial are written on the two layer of carbonless copy paper, but randomnumber and group allocation are only written on the second layer of paper, then placed paper in sealed, opaque, sequentially numbered envelopes. A independent research assistant will conduct the group allocation. Participants are required to write their name and admission number on the first layer of paper after disclose the envelopes, random-number sequence and group allocation can be seen only on the second layer of paper, once two layer of paper is separated, it can't be restored. After the signature of the informed consent, the rater completes the baseline assessment, then the research assistant who is blinded to the study will perform the group allocation. Eligible participants will be randomly divided into either MT group or control group in a ratio of 1:1. After disclose the envelope, the participant who obtains a paper marked with number " 1 " will be allocated to the MT group, otherwise, the participant who obtains a paper marked with"2" will be allocated to the control group. Because of nature of the intervention, rehabilitation therapist and nurse are not blinded, but they are not informed of trial purpose, the rate, research assistant, and statistician are blinded, they are also required not to ask the group and intervention from participants.

\section{Intervention}

Participants are randomly grouped into either MT group or control group. Both groups are administered free range-of-motion upper limb exercise from the first-day post-operation. Free range-of-motion upper limb exercise is the recommendation of the guidelines and specifications for breast cancer diagnosis and treatment developed by the Chinese Anti-Cancer Association, Committee of Breast Cancer Society (2019 edition $)^{14}$. The control group engages in free range-of-motion upper limb exercise in the usual way, while the MT group receives free range-of-motion upper limb exercise based on MT. The intervention instructions will be provided to participants at the time from the admission to surgery, ensuring they are familiar with their exercise. Both groups are supervised and instructed by nurse and rehabilitation therapist. Upon discharge, each participant will be given an A6 envelope containing an exercise log, which is used to record exercise time and frequency, as well as adverse events. Participants are required to bring the exercise log at every assessment for review. In addition, verbal instruction, a written booklet, and videos designed for the intervention will be provided to participants for facilitating intervention at home. and participants in the MT group will be provided with a plane mirror.After discharge, participants are required to exercise at home and will be supervised by intervention staff every Tuesday or Thursday by WeChat or telephone. For participants with poor adherence, the intervention staff will give them verbal encouragement and motivation. Regularly rewarding participants who adhere to exercise(e.g., small juicer, vacuum cup, towel, toothpaste). Intervention staff will share health knowledge to both groups biweekly 
involving diet, self-care, exercise,sexual life for building long-term trust with participants. Both groups are required not to engage in exercise such as arm stretch, Yoga, Pilates, excluding participants reporting morning stiffness at or their shoulder more than 30 minutes. The elastic bandage are also not allowed in intervention. The intervention in both groups are as follows.

Control group

Participants of the control group receive free range-of-motion upper limb exercise from the first day after surgery, as shown in table1.

Table.1 BC Rehabilitation program 


\section{- Preparation phase}

1. If possible, exercise in a quiet room.

2. Do not exercise when you feel dizzy, nauseous, vomiting or have lower back pain.

3.If you take blood pressure medication, exercise after 30 minutes.

4. If your shoulder appears morning stiffness more than 30 minutes please perform 5-10 minutes arm stretch training combined with massage before exercise.

\section{- BC Rehabilitation}

\section{1-2 days after surgery}

a. Please stand relaxed, put your arm in a position with flexed elbows and slightly extended shoulders, then hold a bouncy ball in the palm of your hand to squeeze it as hard for 2-3 seconds, then relax and spread fingers.

b. Arms are positioned in the same as above, then rotate the wrist clockwise and anticlockwise.

Frequency: Exercises are repeated, 10 repetitions a set, 2 sets/twice daily.

Efficacy: promote muscle contraction, blood, and lymph flow, and avoid upper limbs swelling.

\section{3-7days after surgery.}

a. Please stand relaxed, put your arm at your sides, then bring the hand of the affected limb to the ipsilateral and contralateral shoulder alternately while opening and closing the elbows.

b. keep the exercise in stage 1 .

Frequency: 10 repetitions a set, 2 sets/twice daily.

Efficacy: to recover rotation and adduction.

\section{8-14days after surgery.}

a. Please stand, put your arm at your sides, raise the arms forward in a range of $0^{\circ}-120^{\circ}$, perform backward in a range of $0^{\circ}-60^{\circ}$

b. Please stand, put your arm in a position with flexed elbows and slightly extended shoulders( neutral ), then perform abduction and adduction of the shoulder(shoulder in adduction).

c. Please stand, put your arm at your sides, then perform abduction and adduction of the shoulder in a range of $0^{\circ}-120^{\circ}$

d. ** Turn your shoulders forwards and backward

f. ${ }^{* \star}$ please stand in front of the wall, place your palm of affected arm on the wall and run up with moving your finger until you feel can't run up more, maintain for 1 mins, then run down.

Frequency:10 repeats a set, 3 sets $\times$ twice daily

Efficacy: to promote shoulder flexion, abduction, and adduction

\section{2-8weeks after surgery.}

a. Please stand relaxed, put your arm at your sides, raise the arms forward-backward to an active range of motion as most tolerated 
b. Please stand relaxed, your arms were positioned in abduction at shoulder height and flex elbows in 90 degrees( $90^{\circ}$ of abducted position), then rotate the forearms up and down.

c. Please stand relaxed, put your arm at your sides, then perform abduction and adduction of the shoulders to an active range of motion as most tolerated

d. ** please stand the side of your body against a wall, place your palm of affected arm on the wall and run up with moving your finger until you feel can't run up more, maintain for 1 mins, then run down. Keep the "d and $f$ " in stage 3.

Frequency:10 repeats a set, 3 sets $\times$ twice a day, 4 days per week.

Efficacy: stretching and releasing the softness in the anterior chest wall to avoid the chest wall tightness.

\section{- Attention!}

1. Please don't forget the "Preparation phase" before your exercise.

2. It's better not to arbitrarily change the prearranged sequence of exercise for safety and efficacy.

3. At stage 3 , increase the range of motion as tolerated.

4. A muscular glide in the wrist can be seen when squeezing the ball.

5. Don't bend the elbow when you perform flexion and abduction of the Shoulder

6. If the pain caused by exercise persists, please contact the intervention nurse as soon as possible.

Note: ${ }^{*}$ The movement will be performed without MT for mirror therapy.

\section{Mirror group}

Participants of the MT group receive free range-of-motion upper limb exercise based on the MT from the first day after surgery, the program of free range-of-motion upper limb exercise is the same as control group. The standing mirror that is designed for this trial consist of a plane mirror, an adjustable bracket, and two wheels, the mirror has a size of $70 \mathrm{~cm} \times 50 \mathrm{~cm}$ and bracket with a adjustable height in 1.2-2 meter(Fig.2). The MT intervention process is as follows:

1. Preparation stage: MT will be carried out in a separate, quiet room. Participants are required to remove any accessories (including watches, bracelets, rings, hair bands, etc.) on both upper limbs before intervention. Participants choose to sit or stand depending themselves. The standing mirror is placed in front of participants along the midsagittal plane, with the reflective side facing the unaffected limb,the affected limb of participants is hidden behind the mirror. Participants are asked to lean forward slightly to watch the complete reflection of the unaffected limb in the mirror.

2. Induction stage of Visual feedback: Participants will be told to relax and concentrate on the reflection in the mirror and imagine it as the limb on the affected side, and then move their arms optionally for 2 3 mins. This process will be repeated 3 times to ensure forming the visual feedback of participants.

3. Exercise stage: The therapist instructs participants to perform free range-of-motion upper limb 
Exercise as described in table 1. Particiants need watch the reflection in the mirror of their unaffected limb and think of it as their affected upper limb. During the exercise, the participants were asked to concentrate on the reflection in the mirror.

\section{Potential advert events, and exercise adherence}

Nurse needs to regularly record the amount of fluid drainage, observe wound, and measure the arm circumference for monitoring lymphedema. Adverse events include but are not limited to persistent shoulder pain, disruption of the incisional wound, wound bleeding, skin flap necrosis, etc. Any adverse event occurring after the entry into the study of participants will be truthfully recorded. Participants who reports adverse events have right to withdrawing from the trial, surgero and rehabilitation therapist will provied treatmen and evaluation to them. If participants wishes to continue the intervention, we still provided exercise o them. Adherence to exercise is deemed to be attendance of $85 \%$ of planned exercise sessions, which is a total of 261 sets. Adherence will be calculated according to records of the exercise $\log$.

\section{Outcomes measures}

Outcomes will be measured by a trained rater who is blinded to group allocation at baseline(T0), at 2 weeks(T1), 4 weeks(T2), and 8 weeks after surgery(T3). Participant's demographic information and medical history are collected at baseline, the primary and secondary outcomes are as follows:

Primary outcome

To explore the effect of MT on shoulder function, shoulder joint ROM on the affected limb isprimary outcome. An arm goniometer will be used to obtain shoulder ROM measurements including forward flexion, internal rotation, external rotation, and abduction. We followed Struyf's recommendations to conduct the Goniometric shoulder joint ROM measurements ${ }^{28}$. Participants sit with their arms by their sides. Before assessment, participants are asked to move their shoulders and stretch their arms to relaxed muscle, then move the affected arm as possible as they can until they feel tired. Active external rotation and internal rotation are measured in $90^{\circ}$ of horizontal abducted position of shoulder, meanwhile, the rater will press participants' acromion of the scapula to avoid a joint compensatory.

Secondary outcome

Shoulder function scores as measured on the Constant-Murley Score(CMS), which is the most used tool with good reliability and validity for shoulder function scores ${ }^{29}$. The instrument evaluates shoulder function scores from four different domains(pain, daily activity, active range of motion, and strength), among which, pain and daily activity are self-reported subscales, and the other two domains involving objective assessment of shoulder ROM and shoulder adduction strength. The total score of the instrument is 100 , a higher score indicating a better shoulder function. 
For investigating the fear of movement of subjects with musculoskeletal injury, the Tampa Scale of Kinesiophobia is always considered to be a reliable and valid instrument. There has been studies used the Tampa Scale of Kinesiophobia on fear of movement in breast cancer survivors ${ }^{30-32}$.The original Tampa Scale of Kinesiophobia with 17 items was developed in 1991 by Miller et al ${ }^{33}$, one of its' revised version 13-item TSK with a comparable reliability and validity to the original version will be used in this study $^{34}$. The 13-item TSK is a self-report questionnaire with scored on a 4-point Likert-scale range from 1 (strongly disagree) to 4 (strongly agree). The total score of scale ranges between 13 and 52 , higher scores represent stronger fear-avoidance beliefs. Wen Hu has verified the validity and reliability of the Chinese version of the TSK with Cronbach a of 0.778 and reliability of $0.860^{35}$.

The Disabilities of Arm, Shoulder, and Hand Questionnaire (DASH) is a widely used 30-item self-reported questionnaire that will be used to measure the function and symptoms of the affected upper limb in this study. This questionnaire with the level of agreement is rated on a five-point Likert scale, and higher scores indicating a higher amount of problems in arm functioning or symptoms. The questionnaire assesses problems in daily functioning that derives from arm, shoulder, or hand troubles(21 items), abnormal symptoms of the arm(5 items), as well as the effects of arm functional problems on social activities, work, sleep, and psychology(4 items).

Visual Analogue Scale(VAS) is a convenient, simple instrument with a numeric rating scale between 0 and 10 that enables patients to mark their pain intensity on the line. Numerical values from 0-10 represent the pain intensity from no "pain" to "worst pain" correspondingly. In this trial, we assess the pain of participants both in rest and free range-of-motion upper limb exercise.

Hand grip strength will be measured with a Grip Strength Meter. Before test, adjusting unit and years in display panel of Grip Strength Meter. Participant are seated in a chair with her feet on the ground and her knee bending to $90^{\circ}$, then hold the handgrip force meter and slowly pulling the handle as hard as possible. Participants are required to test twice, selecting the highest value as the final record.

Arm circumference will be assessed by a non-stretch tape at styloid and $10 \mathrm{~cm}$ intervals from ulnar styloid up to $40 \mathrm{~cm}$ distally. The difference more than $2 \mathrm{~cm}$ of arm circumference between limbs at any measurement point will be considered as having lymphedema. Incidence of lymphedema is the proportion of participants who develop lymphedema accounts for total participants.

\section{Data collection and management}

The baseline assessment is completed before surgery. The rater conducted the second, the third, and the forth assessment(2 weeks, 4 weeks, and 8weeks after surgery) at the ward or outpatient, because participants will come to hospital for removing the suture and receiving sequent treatment at that time. So that, the rater can measure outcomes face to face with participants, which can decrease the dropout of data as possible. A case report form(CRF) that is identified using participant numbers was designed, the information of group allocation on it is hidden. The rater is required to record all information on the CRF truthfully, timely, accurately. An independent clinical assistant who manages medical history of 
participants needs to check the completeness and accuracy of data at each evaluation, for avoiding modification, a encrypted phone will be used to take a photo of data. We also take effective measures to manage the database. Paper-form data are transcribed in a password-protected computer by two independent data-entry personnel using the input-review mechanism. Final trial data set will be accessed and analyzed by an independent statistician and primary researcher. The data will be upload to the ResMan Raw data sharing platform \IPD sharing platform囚of Chinese Clinical Trial Registry in 6 months upon the completion of the trial. The URL of sharing platform is http://www.medresman.org.cn/login.aspx. The article of this study would be submitted to peer-reviewed journals.

\section{Trial monitoring and quality control}

To assess the fidelity of the intervention and improve the quality of the trial, the study will be surveilled and monitored by the Ethical Committee and research center every 4 months, they are independent of the study and has no competing interests. The content surveilled includes the number of eligible participants, data, exercise logs. The primary investigators need to report the process and problems of the study, and corresponding solutions as well.Interim analysis is required at the mid-term examination, at the final examination, primary researcher will submit test summary, CRF, raw data set to research center.

\section{Data analysis}

Data analysis will be performed by an independent statistician and primary researcher using SPSS24.0 software. ,All statistical tests will be double sides, the statistical significance is set at $p$ value<.05.Means and standard deviation or median and interquartile ranges will be calculated for continuous variables normally distributed and nor-normally distributed respectively. Categorical variables will be described as frequencies(f) and percentages(\%). Demographics and other baseline values will be analyzed using independent two-tailed t-test non-parametric Mann-Whitney U-test, and chi-square test.

This study will adopt the modified intention-to-treat (mITT) principle. The effects of the intervention on primary and secondary outcomes(excluded arm circumference and lymphedema) between groups will be analyzed by Generalized Estimating Equation(GEE) from treatment, time points and the interaction between group and time. Observed values of those outcomes at four assessments time points will be put into GEE, selecting a autocorrelation matrix and the robust standard error estimation method. Taking outcome index as the response variable, the group, time, and interaction of group and time will be incorporated into the model, in addition, selecting radiotherapy, dominant hand, type of surgery as covariates. The model included main effect and interaction effect, paired comparison variables will be selected, and the Least Significant Difference (LSD) method is used to adjust the multiple comparison effect. Arm circumference and incidence of lymphedema will be analyzed at each measurement time point between group using independent two-tailed t-test and chi-square test. In case of missing data, if missing data is completely random, the sample will not be included in the final data analysis.If missing data is incomplete random missing or non-random missing, multiple interpolation will be used to complete missing values. 


\section{Discussion}

This study is a randomized controlled trial, participants in the control group receive free range-of motion upper limb exercise, and participants in the MT group receive free range-of motion upper limb exercise based on MT. The trial aims to explore whether MT can bring gains of the efficacy of free range-of-motion upper limb exercise on shoulder function in patients after breast cancer surgery. BCS always complain of shoulder function complications such as a restricted shoulder ROM, shoulder pain, weakness, and stiffness of shoulder, it adversely affects physical, psychosocial and social domains of function of breast cancer survivors. Despite breast cancer rehabilitation can prevent shoulder function complications, there seems to be an underutilization of rehabilitation. Recently, a few research have shown that MT has the influence on shoulder movement and pain score in patients with shoulder disease. Up to data, there is only one study showed MT can relieve the persistent pain in breast cancer survivors ${ }^{36}$, but the effects of MT on shoulder function has not been reported by article. MT is a bilateral, symmetrical, convenient motor task, free range-of motion upper limb exercise involves both upper limb, given that they have the same performing mode, we will combine MT with free range-of-motion upper limb exercise in this study to observe the effects of a long time intervention of MT on shoulder function in breast cancer survivors. We look forward this randomized controlled trial can provide a successful combination of nurse and rehabilitation, which will positively impact nurse interdisciplinary research. If the study purpose can be examined, MT will be a potential nursing technique for shoulder function in breast cancer survivors.

\section{Study status}

The recruitment of participants started from 1 st July 2020, at the time of the manuscript submission, it is still ongoing. Affected by the epidemic of the new coronavirus, the recruitment is expected to be completed on 31st June 2021, the version number and protocol number: V2.0, 30 April 2021.

\section{Abbreviations}

BC: breast cancer; BCS: breast cancer survivors; ROM: Range of Motion

CRF: case report form; MT: mirror therapy; CMS: Constant-Murley Score;

DASH: Disability of arm-shoulder-hand; TSK: Tampa Scale of Kinesiophobia; VAS: Visual Analogue Scale

\section{Declarations}

\section{Acknowledgements}

We will be very grateful to hospitals and participants who contributed to this research.

\section{Funding}


The study was approved by the 2020 subject competence enhancement project of the School of Nursing, Shanghai University of Traditional Chinese medicine (grant number 2020HLXK06). The fund body is not a participant in any process including study design, data collection, data analysis, or data interpretation of the study.

\section{Authors' Contributions}

$\mathrm{C}-\mathrm{QW}$ designed this study, and she is the person in charge of the research. R-ZY contributed to the writing of this manuscript. K-PL contributes to the quality controlling of the study and assisted in MT intervention. W-Z participated in the design of the study and prepared for ethical approval. Y-Y assisted in the recruitment of participants. X-LW, M-YW, J-TJ assisted in the drafting of this manuscript. All authors have approved the final manuscript.

\section{Availability of data and material}

Data sharing is not applicable to this article at present. The results of the study will be submitted for publication once the data collection and analysis are completed.

\section{Competing interests}

All authors declare that they have no competing interests.

\section{Ethics approval and consent to participate}

This study has been approved by The Ethical Committee of the Longhua hospital affiliated with the Shanghai University of traditional Chinese medicine on 5 May 2020(Approval No. 2020LCSY016). ). All participants will provide written informed consent.

\section{Consent for publication}

Not applicable.

\section{Author details}

${ }^{1}$ School of Nursing, Shanghai University of Traditional Chinese Medicine. ${ }^{2}$ Shanghai East Hospital Tongji University.

${ }^{3}$ School of Rehabilitation, Shanghai University of Traditional Chinese Medicine. ${ }^{4}$ Department of Galactophore, Longhua hospital Shanghai University of Traditional Chinese Medicine. ${ }^{5}$ Department of General Surgery, Fudan University Zhongshan Hospital.

\section{References}


1. Latest global cancer data: Cancer burden rises to 19.3 million new cases and 10.0 million cancer deaths in 2020. https://www.iarc.fr/fr/news-events/latest-global-cancer-data-cancer-burden-rises-to19-3-million-new-cases-and-10-0-million-cancer-deaths-in-2020/

2. Allemani C, Matsuda T, Di Carlo V, et al. Global surveillance of trends in cancer survival 2000-14 (CONCORD-3): analysis of individual records for 37513025 patients diagnosed with one of 18 cancers from 322 population-based registries in 71 countries [J]. The Lancet, 2018, 391(10125): 1023-1075.

3. Stubblefield MD, Keole N. Upper body pain and functional disorders in patients with breast cancer. PM R. 2014;6(2):170-83.

4. Hopkins JO, Allred J, Hurria A, et al. Lymphedema, musculoskeletal events and arm function in older patients receiving adjuvant chemotherapy for breast cancer (Alliance A171302) [J]. Breast cancer research and treatment, 2017, 166(3): 793-808.

5. Verbelen H, Gebruers N, Eeckhout FM, et al. Shoulder and arm morbidity in sentinel node-negative breast cancer patients: a systematic review [J]. Breast cancer research and treatment, 2014, 144(1): 21-31.

6. Hauerslev KR, Madsen AH, Overgaard J, Damsgaard TE, Christiansen P. Long-term follow-up on shoulder and arm morbidity in patients treated for early breast cancer. Acta Oncol. 2020 Jul;59(7):851-858.

7. Dunne M, Keenan K. CE: Late and Long-Term Sequelae of Breast Cancer Treatment. Am J Nurs. 2016;116(6):36-45.

8. Lee CH, Chung SY, Kim WY, Yang SN. Effect of breast cancer surgery on chest tightness and upper limb dysfunction. Medicine (Baltimore). 2019;98(19):e15524.

9. Fourie WJ. Considering wider myofascial involvement as a possible contributor to upper extremity dysfunction a) following treatment for primary breast cancer. J Bodyw Mov Ther. 2008;12(4):349-55.

10. Williams NR, Williams S, Kanapathy M, Naderi N, Vavourakis V, Mosahebi A. Radiation-induced fibrosis in breast cancer: A protocol for an observational cross-sectional pilot study for personalised risk estimation and objective assessment. Int J Surg Protoc. 2019;14:9-13.

11. Lipps DB, Leonardis JM, Dess RT, McGinnis GJ, Marsh RB, Strauss JB, et al. Mechanical properties of the shoulder and pectoralis major in breast cancer patients undergoing breast-conserving surgery with axillary surgery and radiotherapy. Sci Rep. 2019;9(1):17737.

12. Herrera JE, Stubblefield MD. Rotator cuff tendonitis in lymphedema: a retrospective case series. Arch Phys Med Rehabil. 2004;85(12):1939-42.

13. Dinas K, Kalder M, Zepiridis L, Mavromatidis G, Pratilas G. Axillary web syndrome: Incidence, pathogenesis, and management. Curr Probl Cancer. $2019 ; 43(6): 100470$.

14. Breast cancer Professional Committee of Chinese Anti-Cancer Association. CHINA ONCOLOGY 2019;29:609-680.

15. Paolucci T, Bernetti A, Paoloni M, Capobianco SV, Bai AV, Lai C, Pierro L, Rotundi M, Damiani C, Santilli V, Agostini F, Mangone M. Therapeutic Alliance in a Single Versus Group Rehabilitative 
Setting After Breast Cancer Surgery: Psychological Profile and Performance Rehabilitation. Biores Open Access. 2019;8(1):101-110.

16. Stubblefield MD. The Underutilization of Rehabilitation to Treat Physical Impairments in Breast Cancer Survivors. PMR. 2017;9(9S2):S317-S323.

17. Cheville AL, Tchou J. Barriers to rehabilitation following surgery for primary breast cancer [J]. Journal of surgical oncology, 2007, 95(5): 409-418.

18. Ramachandran VS, Rogers-Ramachandran D, Cobb S. Touching the phantom limb [J]. Nature, 1995, 377(6549): 489-490.

19. Altschuler EL, Wisdom SB, Stone L, et al. Rehabilitation of hemiparesis after stroke with a mirror [J]. Lancet (London, England), 1999, 353(9169): 2035-2036.

20. Imaizumi S, Asai T, Koyama S. Agency over Phantom Limb Enhanced by Short-Term Mirror Therapy. Front Hum Neurosci. 2017;4(11):483.

21. Thieme $\mathrm{H}$, Morkisch N, Mehrholz J, et al. Mirror therapy for improving motor function after stroke [J]. The Cochrane database of systematic reviews, 2018;7(7): Cd008449

22. Altschuler EL, Hu J. Mirror therapy in a patient with a fractured wrist and no active wrist extension [J]. Scand J Plast Reconstr Surg Hand Surg, 2008, 42(2): 110-111.

23. Rosen B, Lundborg G. Training with a mirror in rehabilitation of the hand [J]. Scand J Plast Reconstr Surg Hand Surg, 2005, 39(2): 104-108.

24. Louw A, Puentedura EJ, Reese D, Parker P, Miller T, Mintken PE. Immediate Effects of Mirror Therapy in Patients With Shoulder Pain and Decreased Range of Motion. Arch Phys Med Rehabil. 2017;98(10):1941-1947.

25. Başkaya MÇ, Erçalık C, Karataş Kır Ö, Erçalık T, Tuncer T. The effıcacy of mirror therapy in patients with adhesive capsulitis: A randomized, prospective, controlled study. J Back Musculoskelet Rehabil. 2018;31(6):1177-1182.

26. Deconinck FJ, Smorenburg AR, Benham A, Ledebt A, Feltham MG, Savelsbergh GJ. Reflections on mirror therapy: a systematic review of the effect of mirror visual feedback on the brain. Neurorehabil Neural Repair. 2015;29(4):349-361.

27. Rossetti Y, Desmurget M, Prablanc C. Vectorial coding of movement: vision, proprioception, or both? J Neurophysiol. 1995;74(1):457-463.

28. Struyf F, Meeus M. Current evidence on physical therapy in patients with adhesive capsulitis: what are we missing? Clin Rheumatol. 2014;33(5):593-600.

29. Vlaeyen JW, Crombez G. Fear of movement/(re)injury, avoidance and pain disability in chronic low back pain patients. Man Ther. 1999;4(4):187-195.

30. Sulan Zhang, Jiaqi Wang, Juan Zhao. A study on the status quo and influencing factors of postoperatophobia in breast cancer patients. Journal of Nursing Science 2020; 35: 24-27.

31. Kocic M, Stankovic A, Lazovic M, Dimitrijevic L, Stankovic I, Spalevic M, et al. Influence of fear of movement on total knee arthroplasty outcome. Ann Ital Chir. 2015 Mar-Apr;86(2):148-155. 
32. Gencay Can A, Can SS, Ekşioğlu E, Çakcı FA. Is kinesiophobia associated with lymphedema, upper extremity function, and psychological morbidity in breast cancer survivors? Turk J Phys Med Rehabil. 2018;65(2):139-146.

33. Miller RP, Kori SH, Todd DD. The Tampa Scale. Unpublished Report, Tampa; 1991.

34. Weermeijer JD, Meulders A. Clinimetrics: Tampa Scale for Kinesiophobia. J Physiother. 2018;64(2):126.

35. Hu W.

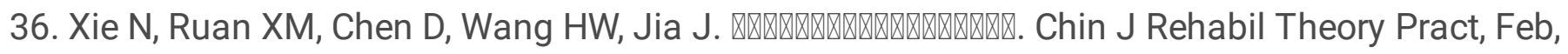
2018;24(2):134-137

37. Chan AW, Tetzlaff JM, Gøtzsche PC, Altman DG, Mann H, Berlin JA, et al. SPIRIT 2013 explanation and elaboration: guidance for protocols of clinical trials. BMJ. 2013;346:e7586.

\section{Figures}

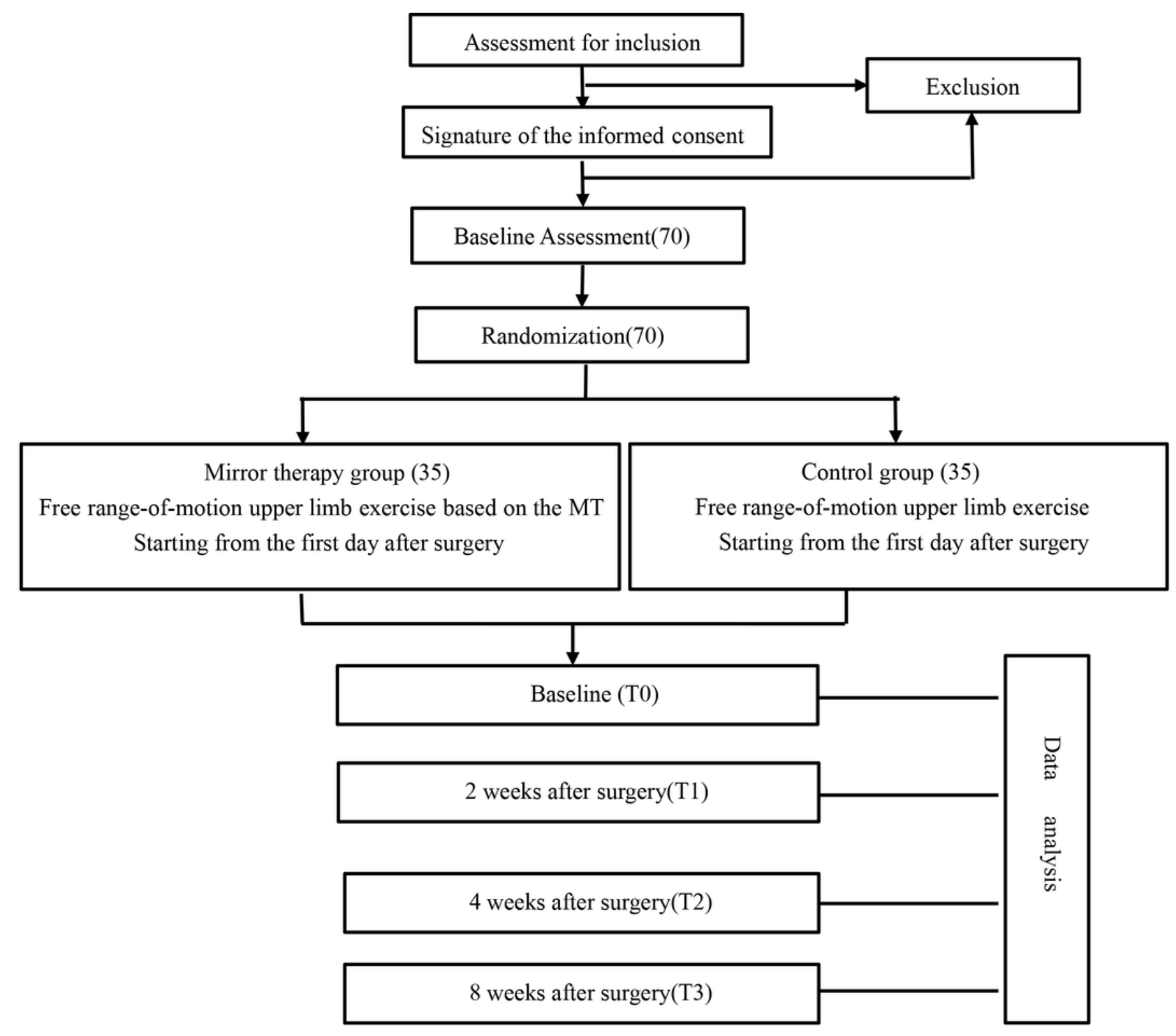


Figure 1

Recruitment and intervention processes flow chart.

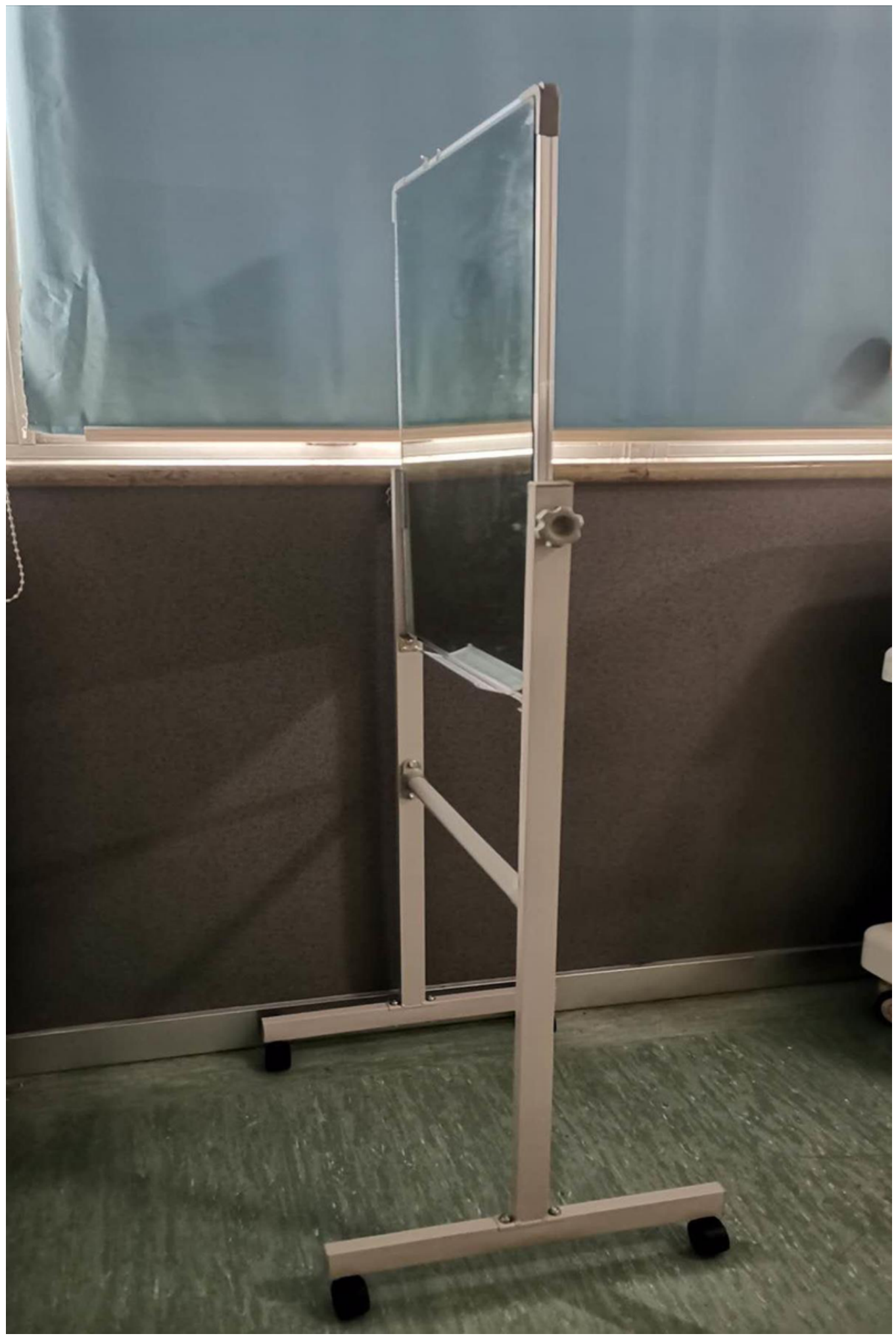

Figure 2

The standing mirror 


\section{Supplementary Files}

This is a list of supplementary files associated with this preprint. Click to download.

- SPIRITChecklist.doc 